\title{
Transport vesicle uncoating: it's later than you think
}

\section{Meg Trahey and Jesse C Hay*}

Address: Division of Biological Sciences and Center for Structural and Functional Neuroscience, The University of Montana, 32 Campus Drive, HS104, Missoula, MT 59812-4824, USA

* Corresponding author: Jesse C Hay (jesse.hay@umontana.edu)

FI000 Biology Reports 2010, 2:47 (doi:10.34I0/B2-47)

The electronic version of this article is the complete one and can be found at: http://fl000.com/reports/biology/content/2/47

\begin{abstract}
Transport vesicle coat proteins play active roles in vesicle cargo sorting as well as membrane deformation and fission during vesicle biogenesis. For years, it was assumed that this was the extent of the coats' function and that the coats depolymerized immediately after vesicle budding, leaving the exposed fusion machinery free to find, dock, and fuse with the proper target membrane. Recently, however, it has become increasingly clear that the coat remains on transport vesicles during their post-budding life and in fact helps properly pair up the vesicle with its intended target membrane. These data have brought up urgent questions about exactly when vesicles do uncoat and how uncoating is regulated. Here, we summarize the latest round of evidence for post-budding roles for coats, including a few hints about how the uncoating process may be coupled to docking and fusion. We also speculate about the possibility of post-fusion functions for residual coats.
\end{abstract}

\section{Introduction and context Vesicular transport}

Intracellular protein transport is, in part, vesiclemediated with biochemically distinct vesicles trafficking along different routes. Three well-studied examples include clathrin-coated vesicles (CCVs) (plasma membrane endocytosis, transport from trans-Golgi network [TGN] to endosomes and lysosomes), coat protein complex II (COPII) vesicles (endoplasmic reticulum [ER] to Golgi), and COPI vesicles (intra-Golgi and Golgi to ER). These diverse vesicles are formed by fundamentally similar processes involving (a) protein cargo selection through cargo receptors; (b) cargo receptor interaction with inner-coat subunits coordinated or monitored by GTPases of the Arf family (COPI vesicles and CCVs formed in the TGN), Sar family (COPII vesicles), or dynamin family (endocytic CCVs); (c) recruitment of outer-coat subunits by the cargo-bound inner coat; (d) membrane curvature; and (e) vesicle scission/budding. Newly budded coated vesicles move (often through interactions with the cytoskeleton), encounter target membranes through specific tethering protein interactions that involve Rab GTPases, and then undergo SNARE (soluble N-ethylmaleimide-sensitive factor attachment protein receptor)-mediated membrane fusion, delivering their cargo to the appropriate target compartment.

\section{The conventional view of uncoating}

A priori, one can imagine that vesicle coats would be a physical impediment to subsequent membrane fusion, so considerable attention has been given to how coats come off. Experiments with COPII coats in the 1990s suggested that, in fact, uncoating takes place soon after budding, leading to an informal consensus that uncoated vesicles are the substrate for tethering and fusion. How did this picture, which now appears to be inaccurate, develop? The formation of a COPII-coated vesicle is initiated by the ER-localized guanine nucleotide exchange factor (GEF) for the Sar1 GTPase (Sec12), which catalyzes the exchange of GDP for GTP on Sar1. This exchange results in a conformational change in Sar1, exposing an amphipathic alpha-helical membrane-binding domain. Membrane-bound Sar1-GTP recruits the inner-coat subunits Sec23/24, which in turn recruit the outer subunits Sec13/31. The yeast COPII coat subunit 
Sec23 displayed GTPase-activating protein (GAP) activity toward Sar1p [1], suggesting that assembly of the coat would lead to activation of the GTPase activity of Sar1 and the resulting Sar1-GDP would trigger coat disassembly. This conclusion was supported by experiments in permeabilized mammalian cells [2], where it was found that if Sar1 GTPase activity was inhibited, the vesicle retained its COPII coat. Real-time monitoring of the coat assembly and disassembly processes using liposomes [3] with purified coat subunits confirmed that, indeed, Sec23 stimulated the GTPase activity of Sar1, which initiated coat disassembly. In addition, the GAP activity of Sec23 activity was itself enhanced 10 -fold by Sec13 binding (i.e., assembly of the outer coat). In the COPI system, GTP hydrolysis by Arf1 was required for coat release and vesicle fusion [4]. Positive membrane curvature (i.e., forming the spherical vesicle) was shown to increase the ability of ArfGAP1 to stimulate the GTPase activity of Arf1 and trigger COPI coat disassembly [5]. Coupled with a lack of any data implying that the coat stays on, all of these findings together naturally led to a model wherein the state of the coat is regulated by the GTP cycle of the small GTPases, which in turn is regulated by associated GEFs and GAPs: in other words, when Sar1 or Arf1 is GTP-bound, the coat comes on, and when it is GDPbound, the coat comes off.

As illustrated in the following sections, newer and more physiological data argue that coats stay on (at least partially) until at least the tethering step. We must therefore amend our view of the meaning of the GTPase cycle in vesicle uncoating. While beyond the focus of the present review, we will briefly outline two additional functions for Sar/Arf GTP hydrolysis that underscore its importance in steps prior to uncoating. These functions are (a) 'proof-reading' the cargo selection process and (b) actuating vesicle fission. The role in cargo selection was suggested by work with cargospiked COPII liposomes and planar membranes $[6,7]$. During formation of prebudding complexes comprised of Sar1-GTP and Sec23/24, the Sec24 subunit is free to interact with transmembrane cargo. In the absence of cargo, Sec23/24 stimulates the GTPase activity of Sar1 and both Sec23/24 and Sar1-GDP are released from membranes. However, in the presence of cargo, Sec23/ 24 remains membrane-associated for multiple rounds of GTP hydrolysis and Sar1 release. Furthermore, multiple rounds of GTP hydrolysis appear to drive accumulation of cargo in prebudding complexes and exclusion of non-cargo molecules. These in vitro experiments are supported by in vivo analysis of COPII subunit turnover at single ER exit sites in living cells [8], where decreasing the available cargo results in a decreased residence time of $\operatorname{Sec} 23 / 24$ on the membrane. A requirement for GTP hydrolysis in the fission of the budded vesicle has been proposed for CCVs [9], COPI vesicles [10], and COPII vesicles [11]. So while the GAP and GTPase activities may play a less central role in uncoating than we thought, they clearly have plenty to do with cargo sorting and vesicle fission.

\section{Major recent advances Coats are retained at least through the initiation of tethering}

Since coats physically interact with cargo proteins, lipids, accessory factors, and themselves, it is not surprising that they can hang on to membranes after vesicle fission. Furthermore, over the last several years, the conventional uncoating model has been challenged by numerous studies demonstrating interactions between coat subunits and tethers or other components of the target membrane. The key advance was the realization, established using in vitro reconstitutions of heterotypic and homotypic COPII vesicle tethering, that these interactions were functionally important for the tethering step per se [12]. This work formed a functional basis for numerous other examples of coat-tether interactions to be interpreted along the same lines. Table 1 summarizes nine instances from work on functionally distinct coated vesicles in both yeast and mammalian systems. The notes are included to highlight particularly strong evidence that the proposed coat-tether interaction is required for the delivery or consumption of transport vesicles. An earlier functional result from permeabilized yeast cells - that excess COPII inner-shell protein Sec23 inhibited fusion but not tethering - is compatible with the apparent functional role of Sec23 in targeting COPII vesicles [13]. Altogether, this literature suggests that the coat is at least partially retained on vesicles in the cytosol and that this residual coat participates in at least the initiation of tethering.

Advances in the last year on the study of yeast retrograde Golgi to ER transport are worthy of extra discussion. At this transport step, COPI vesicles dock and fuse with the ER via the Dsl1 tethering complex. The 2009 papers from this system together make a compelling case that, as shown with COPII coats, COPI coats linger on vesicles in the cytosol and may play an important role in vesicle targeting. First, Schmitt's group [14] mapped multiple interactions between the Dsl1 tether subunit and COPI subunits comprising both the inner and outer shell of the coat. When living yeasts were depleted of the Dsl1 subunit, heavily coated COPI vesicles accumulated, just as would be predicted if the tethering step preceded and was a prerequisite for uncoating. The authors speculated that, in addition to mediating the tethering event itself via COPI binding, the Dsl1p complex might be involved 
Table I. Examples of coat-tether interactions or other coat interactions believed to be required for vesicle targeting

\begin{tabular}{|c|c|c|c|c|c|}
\hline Transport step & System & Coat subunit & Tether & Reference & Notes \\
\hline ER to Golgi & Yeast & Sec23/24 (COPII) & GrhI (GRASP65 ortholog) & {$[16]$} & \\
\hline ER to Golgi & Mammals and yeast & Sec23 (COPII) & Bet3 (TRAPPI) & {$[12]$} & $\begin{array}{l}\text { Sec23-Bet3 interaction is } \\
\text { required for tethering in vitro. }\end{array}$ \\
\hline Retrograde Golgi to ER & Yeast & $\begin{array}{l}\delta-C O P \alpha-C O P \\
(\mathrm{COPI})\end{array}$ & Dsllp (Dsll complex) & {$[14,17]$} & $\begin{array}{l}\text { Dsll depletion leads to } \\
\text { accumulation of heavily coated, } \\
\text { aggregated COPI vesicles. }\end{array}$ \\
\hline Intra-Golgi & Mammals \& yeast & $\gamma \mathrm{I}(\mathrm{COPI})$ & TrsI30 (TRAPPII) & {$[18,19]$} & \\
\hline Retrograde intra-Golgi & Mammals & $\beta-C O P(C O P I)$ & plI5 & [20] & \\
\hline Retrograde intra-Golgi & Mammals & $\beta-\mathrm{COP}(\mathrm{COPI})$ & Cog3p (COG) & [2I] & $\begin{array}{l}\text { Cog3 knockdown leads to } \\
\text { accumulation of untethered, } \\
\text { COPI coated vesicles. }\end{array}$ \\
\hline Golgi to vacuolar lysosome & Yeast & Apl5 (AP-3) & Vps4I (HOPS) & {$[22]$} & $\begin{array}{l}\text { Vps4I depletion causes } \\
\text { accumulation of post-Golgi } \\
\text { AP3-coated vesicles. }\end{array}$ \\
\hline Endosome to TGN & Mammals & Tip47 (cargo adapter) & RhoBTB3 (tether on Golgi?) & [23] & \\
\hline Endosome to TGN & Mammals & SNXI (retromer) & Rab6IPI (tether?) & {$[24]$} & \\
\hline
\end{tabular}

COG, conserved oligomeric Golgi complex; COP, coat protein complex; ER, endoplasmic reticulum; GRASP65, Golgi reassembly stacking protein 65; HOPS, homotypic fusion and vacuole protein sorting complex; TGN, trans-Golgi network; TRAPP, transport protein particle.

in disassembly of the coat at the target membrane. Meanwhile, a structural study on the Dsl1 complex provided a very congruent model of Dsl1-mediated tethering [15]. The Dsl1 complex is shaped like a twolegged tower with the Dsl1 subunit at the peak. Both legs of the tower, which are comprised of subunits Sec39 and Tip20, are anchored at the ER membrane by their interactions with the N-terminal domains of the ER SNAREs Use1 and Sec20, respectively. The Dsl1 subunit positioned at the apex of the tower is presumed to reach a height of $20 \mathrm{~nm}$ above the vesicle surface and contains within it an unstructured, flexible 'lasso' region. The lasso region contains the sites responsible for COPI interactions - perfectly poised to grab passing COPI vesicles and keep them near the ER until all four SNARES (Use1, Sec20, and Ufe1 on the ER, and Sec22 on the vesicle) can assemble a fusogenic four-helix bundle. While elegant, the Dsl1-COPI vesicle tethering model remains to be functionally verified. The recent advances from this system and the others listed in Table 1 [16-24] bring into focus major questions about transport vesicle uncoating: Is the quantity of residual coat required for tethering sufficient to block SNARE engagement or membrane fusion (or both)? If so, what components and events cause release of the 'excess' coat?

\section{Future directions}

Future research will undoubtedly focus on trying to pin down exactly how vesicle uncoating is spatio-temporally regulated. On the one hand, full uncoating cannot precede tethering since multiple tethers apparently rely upon coats as a basis for assembly and specificity. On the other hand, at least some uncoating or coat destabilization must precede trans-SNARE pairing since otherwise the coat would render the SNAREs functionally inaccessible. Clearly, the uncoating process must be regulated.

\section{When and where does uncoating take place?}

Figure 1 is a speculative model for uncoating events and their relationship to tethering and fusion. Vesicle uncoating may occur at one or more of several phases in trafficking. As originally envisioned, uncoating may begin in the cytosol soon after budding (labeled ' 1 '). Cytosolic factors that promote uncoating may include Hsc70s [25-27], Rabs (membrane GTPases that can be recruited from a cytosolic pool) and their effectors [28], and other factors that promote coat post-translational modifications [28-30]. However, the new advances discussed above argue that, although cytosolic uncoating could be significant, it cannot be complete; functional necessity demands that some coat be present during the tethering event itself. Coat stabilizers such as the EF-hand-containing Sec31-binding protein ALG-2 (apoptosis-linked gene 2) could act to counteract the uncoating, retaining more coat to promote tethering, delay fusion, or regulate subsequent membrane-mixing steps [31]. Key questions are how much coat remains during the tethering step and whether this amount is compatible with fusion. Unfortunately, a rigorous quantitative picture of the remaining coat in a tethered but unfused stage is not available. By way of approximation, immuno-isolated mammalian COPII vesicles, the large majority of which were at least tethered (with an unknown but much smaller component also fused), retained close to $60 \%$ of their original Sec23 relative to a fully coated vesicle with a GMP-PNP (5'-guanylyl imidodiphosphate)-locked COPII coat [12,31]. As depicted, the tethering event involves a macromolecular 
Figure I. Model of potential vesicle-uncoating steps

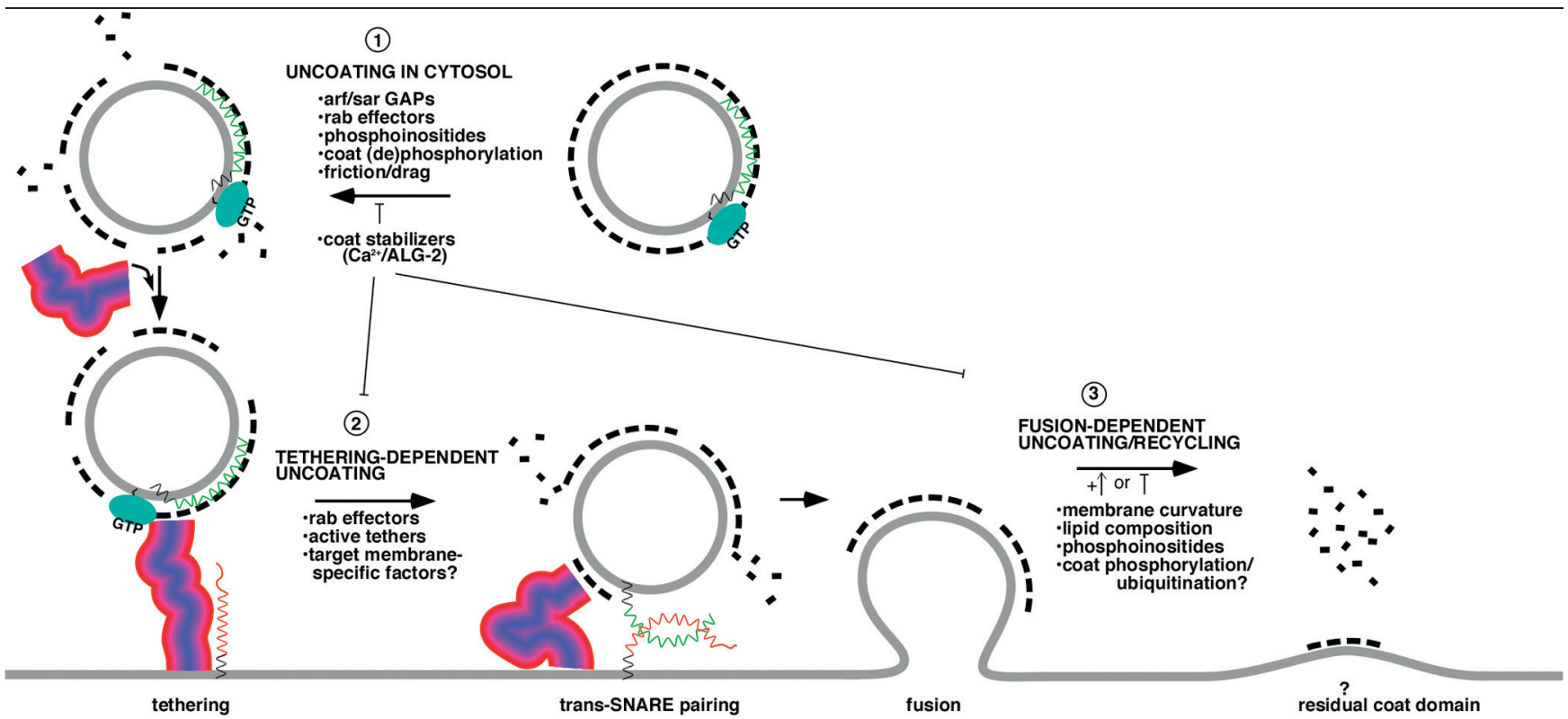

Transport vesicle uncoating may take place in the cytosol prior to tethering ('I'), at the target membrane following tethering ('2'), following fusion (' 3 '), or a combination of these. See the 'When and where does uncoating take place?' section of the text for a detailed explanation. Vesicle coat is represented by heavy dashed lines. A Rab protein is represented by a blue oval. A generic membrane tether (which could be either a multi-subunit tethering complex or extended fibrous tether) is shown as a kinky thick line (magenta with purple core), and SNAREs (soluble N-ethylmaleimide-sensitive factor attachment protein receptors) are shown as helical thin lines. ALG-2, apoptosis-linked gene 2; GAP, GTPase-activating protein.

complex of Rab-GTP, tether, coat, and SNAREs. Suppose that the amount of coat remaining is not compatible with fusion; then perhaps, following the initial culmination of this complex, comes an uncoating event (labeled ' 2 ') in which the coat must be destabilized or shed to a degree necessary to expose the SNAREs, allow close membrane apposition, and provide sufficient membrane fluidity for fusion to be initiated. It makes a lot of sense to posit that the tethering complex itself, or some other focally associated component, initiates this coat depolymerization. For the Dsl1 tethering complex, a complex web of interactions between the Dsl1 subunit and exposed (as well as cryptic) binding sites on the COPI coat has been proposed to initiate a focal depolymerization process [14]. For the transport protein particle (TRAPP)I complex involved in homotypic and heterotypic COPII vesicle fusion, the fact that TRAPPI is a Rab1 GEF and a tether that interacts with the COPII inner layer during tethering suggests a role for Rab effectors in uncoating [12]. Multiple examples (discussed above) now provide evidence that coated vesicles accumulate when tethering is prevented, and yet the nature and directness of tether involvement in uncoating remain a mystery for now. Furthermore, there is no reason to assume that a focally initiated uncoating could cause depolymerization of the entire vesicle coat - most of the coat would be quite distant from the tether site. Whether or not a triggered uncoating step (' 2 ') exists, trans-SNARE pairing and fusion would ensue once the tethered proximal membrane leaflets are sufficiently clear. This would potentially bring about dramatic changes, from the coat's point of view, in membrane curvature, lipid composition, and the availability of cofactors potentially catalyzing coat post-translational modifications [28-30]. The balance of these factors would determine the degree of uncoating occurring during a third, fusion-dependent phase of coat depolymerization (' 3 ').

\section{Is uncoating an all-or-nothing process?}

The recent data and our speculative model of uncoating leave big holes to be dealt with in the future. For example, to what degree do the two coat layers - the inner cargo adaptor layer and the outer-shell layer come off simultaneously as opposed to separately? Do potential phases of uncoating $\left({ }^{\prime} 1{ }^{\prime}, '{ }^{\prime}\right.$ ', or ' 3 ') operate primarily on different layers of coat? To date, there are not enough data on this subject, although one could naturally assume that if the layers uncoat sequentially, then the outer layer should depolymerize first, with the cargo-bound inner layer lingering on the vesicle longer. This order of events has been established as the case for CCVs, for which the outer clathrin layer is removed by 
specific cytosolic factors, leaving bound the cargo adaptor layer to potentially participate in later events $[32,33]$. In addition, we wonder how cooperative the uncoating process is. When an uncoating factor disrupts the coat at a focal spot on the vesicle, would that destabilize the entire coat and necessitate complete depolymerization of the coat? Finally, until proven otherwise, we should remind ourselves not to assume that uncoating goes to completion even after fusion with the target organelle. Remnants of coat could well serve the purpose of defining a domain with similar cargo remaining relatively concentrated, facilitating sorting from cargo with distinct destinations (labeled 'residual coat domain' in the model). An example where such a post-fusion role for remnant coat might make sense would be the vesicular tubular cluster, which is involved in concentrating and distilling COPII-derived anterograde cargo by eliminating most membrane through the generation of retrograde COPI vesicles. Interestingly, immuno-electron tomography revealed free, dumbbellshaped, partially coated COPII-coated carriers - perhaps the fusion products of two COPII vesicles [34]. These dumbbells, which were abundant near ER exit sites, exhibited COPII coat on opposite ends but not at the neck region where membrane fusion may have just occurred. In another recent case, it appeared that residual clathrin present on post-Golgi carriers after fusion with the plasma membrane determined the balance between kiss-and-run and complete exocytosis [35]. Such effects on fusion pore dynamics could have important consequences for the mixture of materials that get secreted (with small molecules preferentially released by kissand-run) and would signify an unexpected post-fusion sorting function for residual coats.

\section{Abbreviations}

$\mathrm{CCV}$, clathrin-coated vesicle; COPI, coat protein complex I or coatomer; COPII, coat protein complex II; ER, endoplasmic reticulum; GAP, GTPase-activating protein; GEF, guanine nucleotide exchange factor; GTP, guanosine triphosphate; SNARE, soluble N-ethylmaleimide-sensitive factor attachment protein receptor; TGN, trans-Golgi network; TRAPP, transport protein particle.

\section{Competing interests}

The authors declare that they have no competing interests.

\section{Acknowledgments}

Research in the authors' laboratory is supported by National Institutes of Health (NIH) grant R01 GM059378 and a supportive environment in an NIH Center of Biomedical Research Excellence (COBRE) (P20 RR015583).

\section{References}

I. Yoshihisa T, Barlowe C, Schekman R: Requirement for a GTPaseactivating protein in vesicle budding from the endoplasmic reticulum. Science 1993, 259:|466-8.

2. Aridor M, Bannykh SI, Rowe T, Balch WE: Sequential coupling between COPII and COPI vesicle coats in endoplasmic reticulum to Golgi transport. J Cell Biol 1995, I 3 1:875-93.

3. Antonny B, Madden D, Hamamoto S, Orci L, Schekman R: Dynamics of the COPII coat with GTP and stable analogues. Nat Cell Biol 200I, 3:53I-7.

FI000 Factor 6.0 Must Read

Evaluated by Catherine Jackson 28 Sep 2001

4. Tanigawa G, Orci L, Amherdt M, Ravazzola M, Helms JB, Rothman JE: Hydrolysis of bound GTP by ARF protein triggers uncoating of Golgi-derived COP-coated vesicles. J Cell Biol I993, | 23: |365-7|.

5. Bigay J, Gounon P, Robineau S, Antonny B: Lipid packing sensed by ArfGAPI couples COPI coat disassembly to membrane bilayer curvature. Nature 2003, 426:563-6.

FI000 Factor 8.2 Exceptional

Evaluated by Karin Romisch 09 Dec 2003, Alfred Wittinghofer 12 Mar 2004, Rainer Pepperkok I4 May 2004

6. Sato K, Nakano A: Dissection of COPII subunit-cargo assembly and disassembly kinetics during Sarlp-GTP hydrolysis. Nat Struct Mol Biol 2005, I 2:167-74.

FI000 Factor 6.0 Must Read

Evaluated by David Stephens 29 Apr 2005

7. Tabata KV, Sato K, Ide T, Nishizaka T, Nakano A, Noji H: Visualization of cargo concentration by COPII minimal machinery in a planar lipid membrane. EMBO J 2009, 28:3279-89.

FI000 Factor 3.0 Recommended

Evaluated by Felix Wieland 10 Nov 2009

8. Forster R, Weiss M, Zimmermann T, Reynaud EG, Verissimo F, Stephens DJ, Pepperkok R: Secretory cargo regulates the turnover of COPII subunits at single ER exit sites. Curr Biol 2006, 16:173-9.

9. Pucadyil TJ, Schmid SL: Conserved functions of membrane active GTPases in coated vesicle formation. Science 2009, 325: $1217-20$.

10. Yang JS, Lee SY, Gao M, Bourgoin S, Randazzo PA, Premont RT, Hsu WW: ARFGAPI promotes the formation of COPI vesicles, suggesting function as a component of the coat. J Cell Biol 2002, I 59:69-78.

FI000 Factor 6.4 Must Read

Evaluated by Rainer Duden 24 Oct 2002, Michael Roth 27 Nov 2002

II. Bielli A, Haney CJ, Gabreski G, Watkins SC, Bannykh SI, Aridor M: Regulation of Sarl NH2 terminus by GTP binding and hydrolysis promotes membrane deformation to control COPII vesicle fission. J Cell Biol 2005, I7I:919-24.

12. Cai H, Yu S, Menon S, Cai Y, Lazarova D, Fu C, Reinisch K, Hay JC, Ferro-Novick S: TRAPPI tethers COPII vesicles by binding the coat subunit Sec23. Nature 2007, 445:94I-4.

FI000 Factor 6.5 Must Read

Evaluated by David Stephens 15 Feb 2007, David K Banfield OI Mar 2007, Volker Haucke 05 Mar 2007

13. Barlowe C: Coupled ER to Golgi transport reconstituted with purified cytosolic proteins. J Cell Biol 1997, I39:1097-I08.

14. Zink S, Wenzel D, Wurm CA, Schmitt HD: A link between ER tethering and COP-I vesicle uncoating. Dev Cell 2009, I 7:403-I6.

FI000 Factor 4.9 Must Read

Evaluated by Anne Spang 20 Oct 2009, David K Banfield 23 Oct 2009, Martin Lowe 27 Oct 2009 
15. Ren Y, Yip CK, Tripathi A, Huie D, Jeffrey PD, Walz T, Hughson FM: A structure-based mechanism for vesicle capture by the multisubunit tethering complex DsII. Cell 2009, 139: I I 9-29.

FI000 Factor 6.6 Must Read

Evaluated by Anne Spang 05 Jan 2010, Martin Lowe II Jan 2010, Josep Rizo 24 Mar 2010

16. Behnia R, Barr FA, Flanagan JJ, Barlowe C, Munro S: The yeast orthologue of GRASP65 forms a complex with a coiled-coil protein that contributes to ER to Golgi traffic. J Cell Biol 2007, |76:255-61.

FI000 Factor 3.2 Recommended

Evaluated by Anne Spang 19 Feb 2007, David Stephens I4 Jun 2007

17. Andag U, Schmitt HD: Dsllp, an essential component of the Golgi-endoplasmic reticulum retrieval system in yeast, uses the same sequence motif to interact with different subunits of the COPI vesicle coat. J Biol Chem 2003, 278:5I722-34.

FI000 Factor 3.0 Recommended

Evaluated by Rainer Duden 18 Dec 2003

18. Cai H, Zhang Y, Pypaert M, Walker L, Ferro-Novick S: Mutants in trs 120 disrupt traffic from the early endosome to the late Golgi. J Cell Biol 2005, I71:823-33.

FI000 Factor 3.0 Recommended Evaluated by Sharon Tooze 02 Feb 2006

19. Yamasaki A, Menon S, Yu S, Barrowman J, Meerloo T, Oorschot V, Klumperman J, Satoh A, Ferro-Novick S: $\mathbf{m T r s} \mathrm{I} 30$ is a component of a mammalian TRAPPII complex, a RabI GEF that binds to COPI-coated vesicles. Mol Biol Cell 2009, 20:4205-15.

20. Guo $Y$, Punj $V$, Sengupta $D$, Linstedt $A D$ : Coat-tether interaction in Golgi organization. Mol Biol Cell 2008, 19:2830-43.

21. Zolov SN, Lupashin VV: Cog3p depletion blocks vesiclemediated Golgi retrograde trafficking in HeLa cells. J Cell Biol 2005, 168:747-59.

FI000 Factor 3.2 Recommended

Evaluated by Catherine Rabouille 04 Mar 2005, Frederick Hughson II Aug 2005

22. Angers CG, Merz AJ: HOPS interacts with Apl5 at the vacuole membrane and is required for consumption of AP-3 transport vesicles. Mol Biol Cell 2009, 20:4563-74.

23. Espinosa EJ, Calero M, Sridevi K, Pfeffer SR: RhoBTB3: a Rho GTPase-family ATPase required for endosome to Golgi transport. Cell 2009, 137:938-48.

24. Wassmer T, Attar N, Harterink M, van Weering IR, Traer C], Oakley J, Goud B, Stephens DJ, Verkade P, Korswagen HC, Cullen PJ: The retromer coat complex coordinates endosomal sorting and dynein-mediated transport, with carrier recognition by the trans-Golgi network. Dev Cell 2009, I 7: I I0-22.

25. Hannan LA, Newmyer SL, Schmid SL: ATP- and cytosoldependent release of adaptor proteins from clathrin-coated vesicles: a dual role for Hsc70. Mol Biol Cell 1998, 9:2217-29.

26. Schmid SL, Braell WA, Rothman JE: ATP catalyzes the sequestration of clathrin during enzymatic uncoating. J Biol Chem 1985, 260: 10057-62.

27. Ungewickell $E$, Ungewickell $H$, Holstein SE, Lindner R, Prasad $K$, Barouch W, Martin B, Greene LE, Eisenberg E: Role of auxilin in uncoating clathrin-coated vesicles. Nature 1995, 378:632-5.

28. Semerdjieva S, Shortt B, Maxwell E, Singh S, Fonarev P, Hansen J, Schiavo G, Grant BD, Smythe E: Coordinated regulation of AP2 uncoating from clathrin-coated vesicles by rab5 and hRME-6. J Cell Biol 2008, 183:499-5II.

29. Cohen M, Stutz F, Dargemont C: Deubiquitination, a new player in Golgi to endoplasmic reticulum retrograde transport. J Biol Chem 2003, 278:51989-92.

30. Dudognon P, Maeder-Garavaglia C, Carpentier JL, Paccaud JP: Regulation of a COPII component by cytosolic O-glycosylation during mitosis. FEBS Lett 2004, 56 I:44-50.

31. Bentley M, Nycz DC, Joglekar A, Fertschai I, Malli R, Graier WF, Hay JC: Vesicular calcium regulates coat retention, fusogenicity, and size of pre-Golgi intermediates. Mol Biol Cell 20I0, 21:1033-46.

32. Greene LE, Eisenberg E: Dissociation of clathrin from coated vesicles by the uncoating ATPase. J Biol Chem 1990, 265:6682-7.

33. Rothman JE, Schmid SL: Enzymatic recycling of clathrin from coated vesicles. Cell 1986, 46:5-9.

34. Zeuschner D, Geerts WJ, van Donselaar E, Humbel BM, Slot JW, Koster A], Klumperman J: Immuno-electron tomography of ER exit sites reveals the existence of free COPII-coated transport carriers. Nat Cell Biol 2006, 8:377-83.

FI000 Factor 8.2 Exceptional

Evaluated by David Stephens 20 Mar 2006, Jesse Hay 05 Apr 2006, Ariel Blocker 12 Apr 2006

35. Jaiswal JK, Rivera VM, Simon SM: Exocytosis of post-Golgi vesicles is regulated by components of the endocytic machinery. Cell 2009, 137:1308-19.

FI000 Factor 8.3 Exceptional

Evaluated by Volker Haucke 08 Jul 2009, Stephen Doxsey 3I Jul 2009. Michael Ehlers 03 Aug 2009, Joachim Seemann 10 Aug 2009, Ruth Collins I4 Aug 2009 